\title{
Coxib plus PPI verhindert Ulzera am besten
}

\author{
Die Kombination eines Coxibs mit einem PPI ist die sicherste NSAR-Therapie. Sie sollte Patienten \\ mit hohem Ulkusrisiko empfohlen werden, wenn sie antiphlogistisch behandelt werden müssen.
}

_ Der Nutzen einer Prophylaxe mit Protonenpumpeninhibitoren (PPI) bei einer Therapie mit nicht-Cox2-selektiven nicht-steroidalen Antirheumatika (nsNSAR) wie Diclofenac oder Ibuprofen ist wissenschaftlich gut fundiert. Der alternative Einsatz eines Coxibs ist weniger oft überprüft worden, und zur Coxib-PPI-Kombination gibt es nur ganz wenige Daten.

$\mathrm{Zu}$ dem Thema wurde nun eine sogenannte Netzwerk-Metaanalyse durchgeführt. Dieses Studiendesign ist ein indirekter Vergleich: Wenn A besser wirkt als B und B besser als C, dann muss auch A besser sein als C. In die Auswertung gingen 82 randomisierte kontrollierte Therapiestudien zu NSAR oder Coxiben mit insgesamt 125.053 Patienten ein. Primäre Endpunkte waren Ulkuskomplikationen (Blutung, Perforation, Stenosen) und symptomatische Ulzera. Sekundäre Endpunkte waren endoskopisch nachgewiesene Ulzera, alle uner- wünschten Ereignisse und Studienabbrüche wegen Nebenwirkungen. Die meisten Studien verglichen nsNSAR mit oder ohne PPI-Prophylaxe.

Ulkuskomplikationen kamen sowohl unter Coxiben als auch unter nsNSAR plus PPI signifikant seltener vor als unter einer nsNSAR-Therapie ohne PPI (relatives Risiko [RR]: 0,25 bzw. 0,28). Unter Coxib plus PPI sank das RR sogar auf 0,07. Auch symptomatische Ulzera wurden durch Einsatz von Coxiben oder nsNSAR plus PPI signifikant seltener. Für diesen Endpunkt fanden sich allerdings keine Studien zur Kombination Coxib plus PPI.

Im sekundären Endpunkt „endoskopisch nachgewiesene Ulzera“ führten sowohl die PPI-Komedikation zu einem nsNSAR als auch der Einsatz eines Coxibs zu einem niedrigeren Risiko als eine nsNSAR-Therapie ohne Prophylaxe. Das niedrigste Risiko fand sich erneut in der Kombination Coxib plus PPI.
Des Weiteren ergab die Analyse, dass nsNSAR plus $\mathrm{H}_{2}$-Blocker der alleinigen nsNSAR-Gabe in Bezug auf Ulkuskomplikationen und endoskopische Ulzera nicht signifikant überlegen war.

- Yuan JQ, Tsoi KK, Yang M. Systematic review with network meta-analysis: comparative effectiveness and safety of strategies for preventing NSAID-associated gastrointestinal toxicity. Aliment Pharmacol Ther. 2016:43:1262-75

\section{KOMMENTAR}

Derartige Netzwerk-Metaanalysen sind interessant, wenn auch Studiendesign, Endpunkte und Studienpopulationen recht homogen sein müssen - was nicht immer automatisch angenommen werden kann. Trotz dieser methodischen Einwände sind die Ergebnisse plausibel und durch die einzelnen Studien untermauert: Die Kombination aus einem Coxib mit einen PPI dürfte das geringste Risiko für eine gastrointestinale UIkuskomplikation haben und kann als NSARTherapie der Wahl bei Patienten mit einem hohen Ulkusrisiko angesehen werden.

Prof. Dr. med. M. Gross

\section{Reines Urat in der Fingerblase}

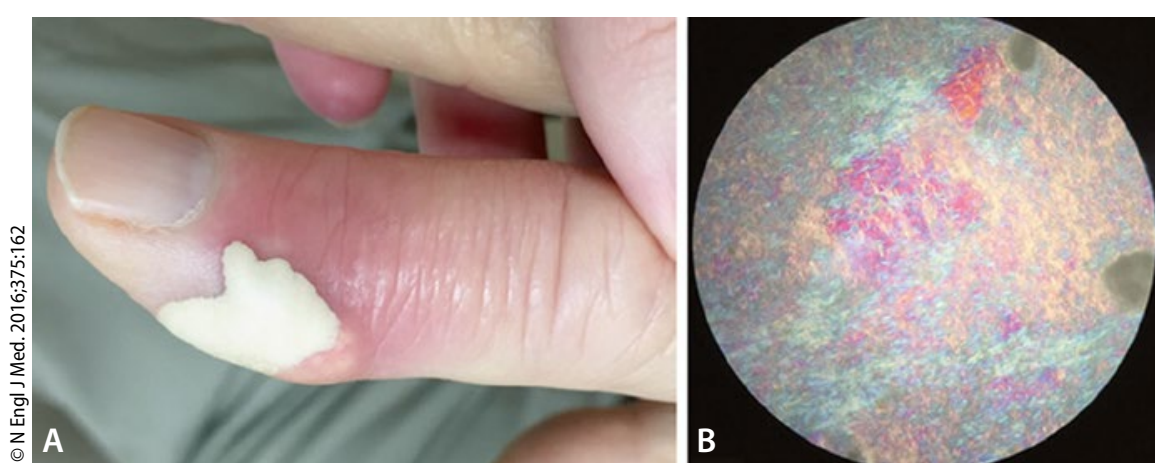

A: Neu aufgetretene Blase am Endgelenk des Zeigefingers. B: Doppelbrechende Uratkristalle im polarisierten Licht.

Ein 71-jähriger Mann mit langjähriger Gichtanamnese und Zustand nach Nierentransplantation war beunruhigt wegen einer neu aufge- tretenen Blase am Endgelenk des rechten Zeigefingers, die etwas distal eines vorbestehenden Tophus aufgetreten war (Abb. A). Ein
Trauma in diesem Bereich war dem Patienten nicht erinnerlich. Aus der Blase entleerte sich eine krümelige, weißliche Flüssigkeit, in der sich im polarisierten Licht doppelbrechende Uratkristalle zeigten (Abb. B). Der Patient wurde mit Allopurinol behandelt, wobei die Dosis schrittweise von 100 auf $300 \mathrm{mg} / \mathrm{d}$ erhöht wurde, bis die Harnsäurekonzentration im Serum auf unter $5 \mathrm{mg} / \mathrm{dl}$ eingestellt war.

Tophi sind ein häufiger Befund bei ungenügend behandelter Gicht; derartige Befunde mit "UratMilch" gefüllter Blasen sind allerdings selten. Es handelt sich dabei um eine Blickdiagnose, die Bestätigung durch mikroskopische Untersuchung im polarisierten Licht ist nicht unbedingt erforderlich.

Prof. Dr. med. H. S. FüeßI

- Robbins RC, Edison JD (rachel.robbins@yahoo.com): Milk of urate bulla. N Engl J Med. 2016;375:162 